\title{
LVSEP Analysis of Cationic Analytes in Cationic Polymer-Coating Microchannel Prepared by Vacuum-Drying Method
}

\author{
Fumihiko KITAGAWA*, Yuka NIIMIYA, Isoshi NUKATSUKA \\ Department of Frontier Materials Chemistry, Graduate School of Science and Technology, Hirosaki University, 3 Bunkyo-cho,
}

Hirosaki 036-8561, Japan

\begin{abstract}
To improve the detection sensitivity of cationic analytes with simple experimental procedures in microchip electrophoresis (MCE), large-volume sample stacking with an electroosmotic flow (EOF) pump (LVSEP) was performed in polymer-coated microchannels. At first, cationic polybrene (PB) was employed as the coating polymer to reverse the EOF. In the LVSEP analyses, however, the PB-coated microchannel gave insufficient enrichments and broader peaks for cationic standard dyes. By the application of neutral poly(vinyl alcohol) and cationic poly(allylamine) mixture-coating, on the other hand, good enrichments of histamine were achieved by LVSEP with a sensitive enhancement factor of 120 .
\end{abstract}

Keywords: Microchip electrophoresis; On-line sample preconcentration; LVSEP; Vacuum-drying coating; Cationic analytes

\section{Introduction}

Microchip electrophoresis (MCE) is a versatile technique especially for biological and pharmaceutical analyses [1-3]. In MCE, rapid and efficient separations can be performed with low sample consumptions. However, the detection sensitivity is often insufficient due to a small amount of a sample. To overcome the sensitivity problem, various on-line sample preconcentration techniques, including stacking [46], sweeping [7,8], selective sweeping [9-11], dynamic pH junction [12], transient isotachophoresis [13-15] and their modified techniques [16-20], have been developed in capillary electrophoresis (CE) and MCE. Among these techniques, large-volume sample stacking with an electroosmotic flow (EOF) pump (LVSEP) has several unique characteristics, e.g., efficient enrichments by a whole column sample-injection, simple experimental procedures based on a voltage application without polarity switching, and high resolutions according to the movement of the stacked analytes zone from the column inlet to the outlet [2130]. However, the LVSEP technique has been mainly applied to the analyses of anionic compounds, i.e., the applications to cations have been limited since a reversal EOF is required. To our knowledge, the MCE-LVSEP analysis of cationic compounds has not been reported.

Figure 1 shows a schematic diagram of LVSEP for cationic analytes. At first, the microchannel is coated with cationic polymers to obtain weakly and positively-charged surface (Fig. 1a) $[21,26]$. A low-ionic strength (I) sample solution containing cationic analytes is fully injected into the channel (Fig. 1b). When a normal polarity voltage is applied, the analytes are concentrated by the difference in the $I$ between the sample matrix and the background solution (BGS). Since the low- $I$ sample solution enhances the anodic EOF velocity $\left(v_{\mathrm{e}, \mathrm{S}}\right)$ in the microchannel, the stacked analytes move to the anode. As the BGS is introduced from the outlet reservoir into the channel (Fig. 1c), $v_{\mathrm{eo}, \mathrm{S}}$ is gradually decreased and finally suppressed due to the high- $I$ BGS (Fig. 1d). Because the electrophoretic velocity $\left(v_{\mathrm{ep}}\right)$ becomes faster than $v_{\mathrm{eo}, \mathrm{BGS}}$, the stacked analytes zone migrates to the cathodic reservoir (Fig. 1e). Hence, the LVSEP technique requires a faster and slower EOF toward the anode at the stacking and the separation step, respectively.

In our previous study, a vacuum-drying method was developed to immobilize neutral and charged polymers for controlling EOF on poly(dimethyl siloxane) (PDMS)-glass hybrid microchips [31]. This coating technique needs only

Received: 19 March 2021

Accepted: 9 April 2021

J-STAGE Advance Published: 21 April 2021

DOI: $10.15583 /$ jpchrom.2021.008
Tel: +81-172-39-3946; Fax: +81-172-39-3946

E-mail: kitagawa@hirosaki-u.ac.jp 
(a) Cationic polymer coating by vacuum-drying method

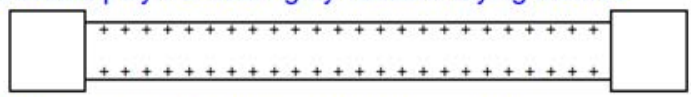

(b) Whole-channel injection of cationic analytes

\begin{tabular}{|c|c|}
\hline $\begin{array}{l}\text { cationic } \\
\text { analytes } \\
\text { in low-I } \\
\text { matrix }\end{array}$ & $\begin{array}{l}++++++++++++++++++++++++ \\
+++++++++++++++++++++++++\end{array}$ \\
\hline
\end{tabular}

(c) Sample stacking and sample matrix removal

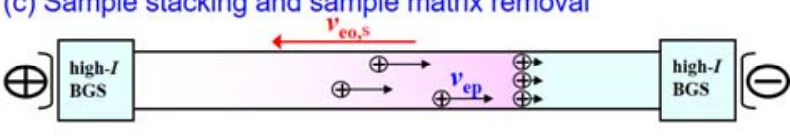

(d) EOF suppression and moving-direction reversal

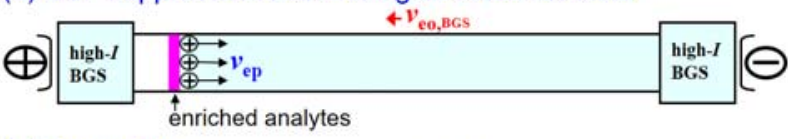

(e) Separation

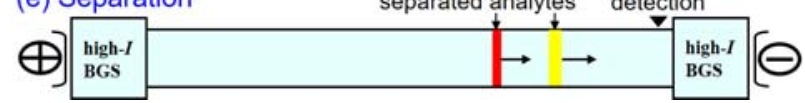

Fig. 1. Schematics of the LVSEP analysis for cationic analytes in the cationic polymer-coated microchannel.

the loading of polymer solutions into the microchannel which is incubated with a sodium dodecyl sulfate (SDS) solution for several minutes to hydrophilize the channel surface, and subsequent vacuum-drying for $10 \mathrm{~min}$. By employing the vacuum-drying method, it is expected that weakly and positively-charged microchannel can be prepared with the simple and rapid experimental procedures. In this study, cationic polymers-coating microchannels prepared by the vacuum-drying technique were applied to the LVSEP analyses of cations.

\section{Experimental}

\subsection{Chemicals}

Hexadimethrine bromide (polybrene (PB), P.N. 107686), poly(allylamine hydrochloride) (PAA, Mw 17,500), poly(vinyl alcohol) (PVA, Mw 80,000, 88\% hydrolyzed) and rhodamine 123 (R123) were obtained from Sigma-Aldrich (Tokyo, Japan), 4-Fluoro-7-nitrobenzofurazan (NBD-F) from DOJINDO (Kumamoto, Japan), PDMS base and curing reagent (Sylgard 184) from Dow Corning Toray (Tokyo, Japan), and SU8-50 from MicroChem (Newton, MA, USA). Rhodamine B (RB), histamine (HA), DL-histidine (His), SDS and all other reagents were purchased from FUJIFILM Wako Pure Chemical (Osaka, Japan). All solutions were prepared with deionized water purified by Auto-Still (WG510, Yamato Scientific, Tokyo, Japan) and Simplicity-UV (Merck Millipore, Billerica, MA, USA) systems, and filtered through a $0.45 \mu \mathrm{m}$ pore membrane filter prior to use.

For fluorescence labeling, $0.5 \mathrm{~mL}$ of $20 \mathrm{mM}$ HA or His in $20 \mathrm{mM}$ borate buffer ( $\mathrm{pH}$ 8.0) containing $5 \mathrm{mM}$ EDTA was mixed with $0.5 \mathrm{~mL}$ of $50 \mathrm{mM}$ NBD-F acetonitrile solution. The mixture was heated to $60^{\circ} \mathrm{C}$ for $3 \mathrm{~min}$, and then cooled at $0^{\circ} \mathrm{C}$ to quench the labeling reaction [32]. The obtained stock solution of NBD-labeled HA or His was stored at $20^{\circ} \mathrm{C}$. In the MCE analysis, the stock solution was diluted to an appropriate concentration with deionized water.

A PDMS microchannel was fabricated by the conventional soft lithography technique. A straight-channel chip had a single channel $(150 \mu \mathrm{m}$ width $\times 100 \mu \mathrm{m}$ depth $)$ with a total separation channel length of $30.0 \mathrm{~mm}$. After producing reservoir wells $(2.5 \mathrm{~mm}$ diameter $)$ with a piercer, these microchannels were directly bonded onto the slide-glass lids $(76.2 \times 25.4 \mathrm{~mm})$ via plasma treatment (YHS-R, SAKIGAKE-Semiconductor, Kyoto, Japan). Fabricated PDMS-glass hybrid microchannels were coated with $\mathrm{PB}$ or PVA+PAA mixture to reverse the EOF by the vacuumdrying method [31]. Briefly, a $10 \mathrm{mM}$ SDS solution was manually introduced into the whole microchannel by using a microsyringe to hydrophilize the PDMS surface. After removing the SDS solution, the microchannel was loaded with the coating polymer solution. The microchip was put into a vacuum-chamber under reduced-pressure $(<\sim 1$ Torr $)$ with an oil rotary vacuum pump (G-50DA, ULVAC KIKO, Saito, Japan) for $10 \mathrm{~min}$. The polymer-coated channel was washed with water, and finally conditioned with the BGS prior to use.

\subsection{MCE measurements}

MCE experiments were performed on a fluorescence microscope (IX73, Olympus, Tokyo, Japan). A 130 W mercury lamp (SHI-1300L, Olympus) for excitation was introduced to the microscope, and irradiated to the microchannel through a dichroic filter (BP460-480GFP, Olympus) and an objective lens (LUCPLFLN 20×, Olympus). Fluorescence from analytes collected by the same objective lens was passed through a dichroic filter (U-FBWA, Olympus), and led to a multichannel photodetector (PMA-12, Hamamatsu Photonics, Hamamatsu, Japan). Microchips were fixed on the microscope stage by using a jig for adjusting two platinum wires, which would ensure the repeatable positioning of the electrodes. All the analytes were detected by the fluorescence detection scheme at the point $5.0 \mathrm{~mm}$ from the cathodic end of the straight channel.

In the LVSEP analysis on the straight-channel chip, a sample solution was introduced into the entire channel by manually using a syringe. The inlet and outlet reservoirs were filled with $5.0 \mu \mathrm{L}$ of a BGS. A voltage of $+1.0 \mathrm{kV}$ was applied with a high voltage power supply (HCZE-30PN0.25, Matsusada Precision, Kusatsu, Japan) through two platinum electrodes immersed in the two reservoirs. To evaluate the enrichment efficiency in LVSEP, the value of sensitive enhancement factor (SEF) was calculated by comparing the peak height obtained under the LVSEP condition with the fluorescence intensity from the microchannel filled with the sample solution (no preconcentration) taking into account the dilution factor. 
To determine the EOF velocity in the polymer-coated microchannels, RB was used as an EOF marker [33]. RB has a carboxylate with the $\mathrm{p} K_{\mathrm{a}}$ value of 3.1 and a protonated diethylammonium groups, so that the electrophoretic mobility of RB is nearly zero in the solution at the $\mathrm{pH}$ value above 5, which is an essential property for the EOF marker. In this study, the value of $\mathrm{pH}$ for the EOF measurement was set at 5.0. On the other hand, the difference in the EOF mobility between in a low- $I$ aqueous solution-filled channel $\left(\mu_{\mathrm{eo}, \mathrm{S}}\right)$ which is almost identical with that in the stacking step (Fig. 1c) and in a high- $I$ BGS-filled channel $\left(\mu_{\mathrm{eo}, \mathrm{BGS}}\right)$ which should agree with that in the separation step (Fig. 1d-1e) is one of the most significant factors to determine the SEF in the LVSEP technique. For the measurements of $\mu_{\mathrm{eo}, \mathrm{S}}$ and $\mu_{\mathrm{e}, \mathrm{BGS}}, 1 \times 10^{-5} \mathrm{M} \mathrm{HCl}(\mathrm{pH} 5.0)$ and $20 \mathrm{mM}$ acetate buffer $(\mathrm{pH}$ 5.0) were loaded in the microchannel, respectively (Fig. 2). The inlet reservoir was filled with $0.1 \mathrm{ppm} \mathrm{RB}$, and then a voltage of $-1.0 \mathrm{kV}$ was applied. Due to the reversal EOF generated in the coating channel, RB molecules were transported to the anodic side. In the EOF experiments, $\mu_{\mathrm{eo}, \mathrm{S}}$ and $\mu_{\mathrm{eo}, \mathrm{BGS}}$ were determined from the migration time.

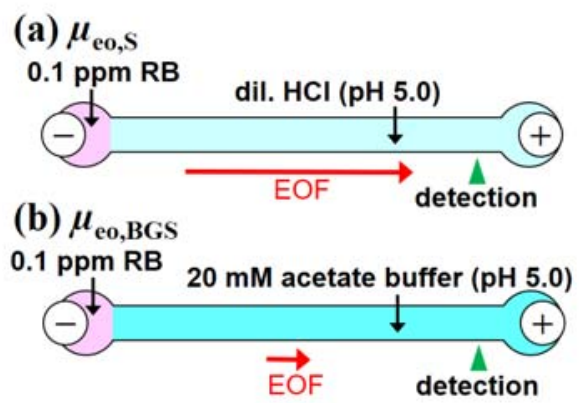

Fig. 2. Schematic illustration of the EOF rate measurements for (a) $\mu_{\mathrm{eo}, \mathrm{S}}$ and (b) $\mu_{\mathrm{eo}, \mathrm{BGS}}$.

\section{Results and discussion}

For the enrichment of cations by LVSEP, a weakly and positively charged surface is required to reverse the EOF $[21,26]$. In our previous study, PB was stably immobilized by the vacuum-drying method [31] to give a reversal EOF. In the preliminary experiments, R123 were employed as a standard cationic dye. Fig. 3 shows LVSEP analyses of R123

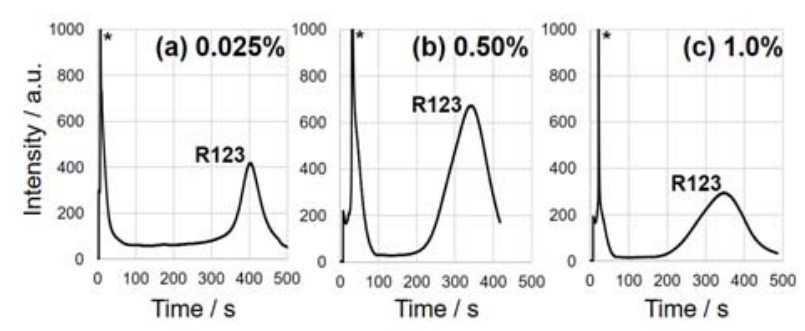

Fig. 3. LVSEP analyses of $0.10 \mathrm{ppm}$ R123 in the PB-coated microchannels. Coating polymer concentrations, (a) 0.025, (b) 0.50 and (c) $1.0 \% \mathrm{~PB}$; BGS, $10 \mathrm{mM}$ phosphate buffer (pH 3.0). Asterisk indicates a system peak in LVSEP.
Table 1. LVSEP analyses of R123 in the PB-coated channel.

\begin{tabular}{cccc}
\hline$[\mathrm{PB}] / \%$ & $\begin{array}{c}\text { migration } \\
\text { time } / \mathrm{s}\end{array}$ & peak width / s & SEF \\
\hline 0.025 & $396(14.6)$ & $60.1(28.1)$ & $54(98.8)$ \\
0.5 & $344(4.2)$ & $131(30.5)$ & $36(34.3)$ \\
1.0 & $347(5.2)$ & $188(36.2)$ & $31(18.9)$ \\
\hline
\end{tabular}

Values in parentheses are \%RSDs $(n=3)$.

Table 2. Electroosmotic data in the PB-coated channel

\begin{tabular}{ccc}
\hline$[\mathrm{PB}] / \%$ & $\mu_{\mathrm{eo}, \mathrm{BGS}} / \mathrm{cm}^{2} \cdot \mathrm{V}^{-1} \cdot \mathrm{s}^{-1}$ & $\mu_{\mathrm{eo}, \mathrm{S}} / \mathrm{cm}^{2} \cdot \mathrm{V}^{-1} \cdot \mathrm{s}^{-1}$ \\
\hline 0.025 & $7.2 \times 10^{-5}(16.4)$ & $1.3 \times 10^{-4}(25.1)$ \\
0.5 & $6.9 \times 10^{-5}(20.2)$ & $1.3 \times 10^{-4}(19.7)$ \\
1.0 & $5.7 \times 10^{-5}(21.9)$ & $1.4 \times 10^{-4}(51.2)$ \\
\hline
\end{tabular}

Values in parentheses are \%RSDs $(n=3)$.

in the PB-coated microchannels with varying the $\mathrm{PB}$ concentration from 0.025 to $1.0 \%$ in the vacuum-drying coating solution. As can be seen, all the electropherograms gave broader peaks of R123. Table 1 summarizes the electrophoretic data for R123 in the PB-coated channel. Although the SEFs were acceptable relative to those in our previous LVSEP report for cations [21], the values of the migration times and the peak widths were quite larger.

To elucidate the reason for this band broadening of the enriched R123 zone in the PB-coated channel, the EOF measurements were carried out as summarized in Table 2 . Prior to the discussion, it should be noted that the electrophoretic mobility of R123 $\left(\mu_{\mathrm{ep}, \mathrm{R} 123}\right)$ was determined to be $1.3 \times 10^{-4} \mathrm{~cm}^{2} / \mathrm{V} \cdot \mathrm{s}$. At the PB concentration of $0.025 \%$, which gave the longest migration time in Table 1 , the $\mu_{\mathrm{eo}, \mathrm{S}}$ was comparable with $\mu_{\mathrm{ep}, \mathrm{R} 123}$. This should cause a quite slower transportation of the enriched R123 zone toward the anode in the stacking step (Fig. 1c), resulted in the longer migration time and the broader peak. At the $1.0 \% \mathrm{~PB}$ concentration, furthermore, the $\mu_{\mathrm{eo}, \mathrm{S}}$ was almost unchanged $\left(1.4 \times 10^{-4} \mathrm{~cm}^{2} / \mathrm{V} \cdot \mathrm{s}\right)$. Thus, the PB-coated channel was not suitable for the LVSEP enrichment of cationic R123 mainly due to slow $\mu_{\mathrm{eo}, \mathrm{S}}$, which would be caused by the insufficient amount of the immobilized PB onto the channel surfaces.

To obtain more suitable channel surfaces for preconcentrating cationic analytes, the application of the PVA+PAA coating, which could enrich aromatic amines by LVSEP in CE [26], was investigated. In the PB-coated channel, the electrostatic repulsion force between the quaternary ammonium groups of the PB molecules would reduce the immobilized amount of the cationic polymers. In the PVA+PAA coating, on the other hand, the amount of the

Table 3. Electroosmotic data in the PVA+PAA -coated channel.

\begin{tabular}{cccc}
\hline$[\mathrm{PVA}]$ & {$[\mathrm{PAA}]$} & $\mu_{\mathrm{eo}, \mathrm{BGS}} / \mathrm{cm}^{2} \cdot \mathrm{V}^{-1} \cdot \mathrm{s}^{-1}$ & $\mu_{\mathrm{eo}, \mathrm{S}} / \mathrm{cm}^{2} \cdot \mathrm{V}^{-1} \cdot \mathrm{s}^{-1}$ \\
\hline $0.90 \%$ & $0.10 \%$ & $9.7 \times 10^{-5}(8.1)$ & $2.1 \times 10^{-4}(23.0)$ \\
$0.95 \%$ & $0.05 \%$ & $1.0 \times 10^{-4}(7.1)$ & $1.9 \times 10^{-4}(20.1)$ \\
$0.99 \%$ & $0.01 \%$ & $1.0 \times 10^{-4}(16.3)$ & $1.8 \times 10^{-4}(10.4)$ \\
\hline
\end{tabular}

Values in parentheses are \%RSDs $(n=3)$. 


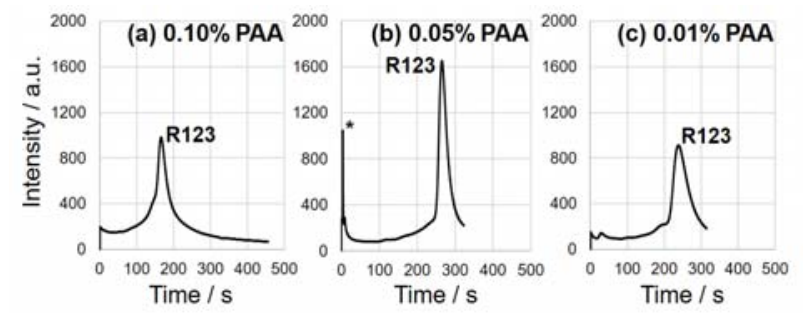

Fig. 4. LVSEP analyses of 0.10 ppm R123 in the PVA+PAAcoated microchannels. Coating polymer concentrations, (a) $0.90 \%$ PVA $+0.10 \%$ PAA, (b) $0.95 \%$ PVA $+0.05 \%$ PAA and (c) $0.99 \%$ PVA $+0.01 \%$ PAA. Other conditions are as in Fig. 3 .

Table 4. LVSEP analyses of R123 in the PVA+PAA-coated channel.

\begin{tabular}{ccccc}
\hline$[\mathrm{PVA}]$ & {$[\mathrm{PAA}]$} & $\begin{array}{c}\text { migration } \\
\text { time / s }\end{array}$ & $\begin{array}{c}\text { peak width / } \\
\mathrm{s}\end{array}$ & SEF \\
\hline $0.90 \%$ & $0.10 \%$ & $168(10.0)$ & $27.1(36.8)$ & $75(24.4)$ \\
$0.95 \%$ & $0.05 \%$ & $251(35.9)$ & $25.8(31.1)$ & $97(38.2)$ \\
$0.99 \%$ & $0.01 \%$ & $254(29.6)$ & $58.0(27.9)$ & $87(35.8)$ \\
\hline
\end{tabular}

Values in parentheses are \%RSDs $(n=3)$.

modified cationic polymers can be increased since PAA consists only of the first-class amine groups. In the coating by the neutral and cationic polymers mixture, the mixing ratio of PVA and PAA is a significant factor for determining the EOF rates and the LVSEP efficiencies. In our previous study [26], the PAA concentration ranging from 0.01 to $0.10 \%$ was suitable to form the weakly and positivelycharged surface for LVSEP. Furthermore, clogging of the microchannel was sometimes problematic at higher PVA concentrations above $1 \%$. Hence, the total concentrations of the polymers were set at $1.0 \%$ with $0.01 \sim 0.10 \%$ PAA. Table 3 shows the EOF data in the PVA+PAA-coated microchannel prepared by the vacuum-drying method. Comparing with Table 2 , the values of $\mu_{\mathrm{e}, \mathrm{S}}$ in the PVA+PAA coating channel were improved to $1.8 \sim 2.1 \times 10^{-4} \mathrm{~cm}^{2} / \mathrm{V} \cdot \mathrm{s}$, which were larger than $\mu_{\mathrm{ep}, \mathrm{R} 123}\left(1.3 \times 10^{-4} \mathrm{~cm}^{2} / \mathrm{V} \cdot \mathrm{s}\right)$. Faster $\mu_{\mathrm{eo}, \mathrm{S}}$ should allow an efficient removal of the vacant sample matrix in the stacking step without band broadening of the enriched analytes zone. On the other hand, $\mu_{\mathrm{eo}, \mathrm{BGS}}\left(1.0 \times 10^{-}\right.$ ${ }^{4} \mathrm{~cm}^{2} / \mathrm{V} \cdot \mathrm{s}$ ) were slower than $\mu_{\mathrm{ep}, \mathrm{R} 123}$. Hence, it was expected that the band-broadening of the enriched cations zone could be suppressed, improving the SEFs in the PVA+PAA-coated microchannel.

Figure 4 shows the electropherograms in the LVSEP analyses of R123 in the PVA+PAA-coated channels, and their analytical results are summarized in Table 4. Relative to Fig. 3, the peak widths of R123 were apparently decreased in the PVA+PAA coatings. As expected from the EOF data in Table 3, the PVA+PAA coatings gave faster transportation in the stacking step, resulting in the shorter migration times, the narrower peak widths and the higher SEFs. However, the peak widths of R123 were still broader, which should be disadvantageous for the separation. This might be caused by

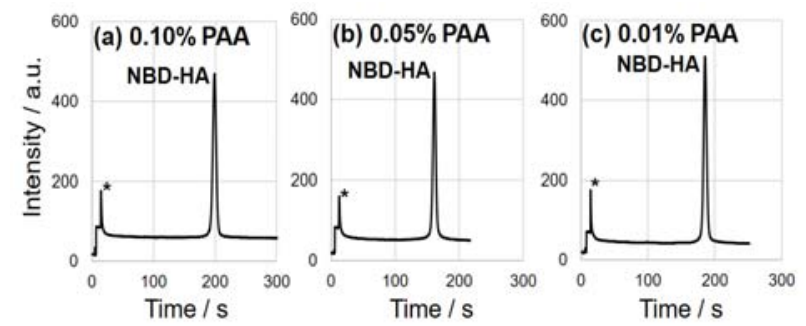

Fig. 5. LVSEP analyses of $5.0 \mu \mathrm{M}$ NBD-HA in the PVA+ PAA-coated microchannels. Conditions are as in Fig. 4.

Table 5. LVSEP analyses of NBD-HA in the PVA+PAAcoated channel.

\begin{tabular}{ccccc}
\hline$[\mathrm{PVA}]$ & {$[\mathrm{PAA}]$} & $\begin{array}{c}\text { migration } \\
\text { time } / \mathrm{s}\end{array}$ & $\begin{array}{c}\text { peak width / } \\
\mathrm{s}\end{array}$ & SEF \\
\hline $0.90 \%$ & $0.10 \%$ & $178(10.0)$ & $5.9(25.4)$ & $95(27.0)$ \\
$0.95 \%$ & $0.05 \%$ & $167(6.4)$ & $5.6(6.6)$ & $113(16.5)$ \\
$0.99 \%$ & $0.01 \%$ & $186(13.3)$ & $6.0(18.2)$ & $120(10.5)$ \\
\hline
\end{tabular}

Values in parentheses are \%RSDs $(n=3)$.

Table 6. LVSEP analyses of NBD-HA and His in the PVA+PAA-coated channel.

\begin{tabular}{cccc}
\hline & migration time / s & SEF & $R \mathrm{~s}$ \\
\hline HA & $178(29.5)$ & $83(19.9)$ & 0.71 \\
His & $189(28.9)$ & $87(12.6)$ & $(52.2)$ \\
\hline
\end{tabular}

Values in parentheses are \%RSDs $(n=3)$. [PVA] $=0.99 \%$, $[\mathrm{PAA}]=0.01 \%$.

the adsorption of rhodamine dyes onto the microchannel [34] even after the PVA+PAA coating.

Instead of R123, NBD-HA was employed as the standard analyte for the LVSEP analysis of cations. As shown in Fig. 5 , quite sharp peaks for the enriched NBD-HA appeared in the PVA+PAA-coated channel mainly due to negligible surface adsorption of both NBD and HA moieties. As a result, the peak width became narrow from 26 58 to $6 \mathrm{~s}$ and the SEFs were improved to $95 \sim 120$. Finally, the proposed method was applied to the separation of NBD-HA and NBDHis, which are important for medical and food analyses since His molecules are metabolized to form allergic HA. In the separation of NBD-HA and His, $0.99 \%$ PVA $+0.01 \%$ PAA

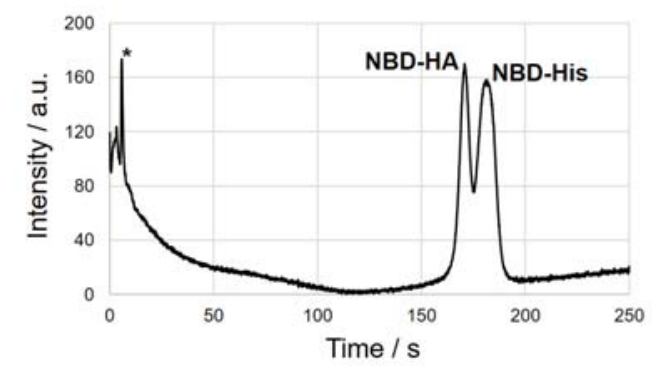

Fig. 6. LVSEP analysis of $0.5 \mu \mathrm{M}$ NBD-HA and $5 \mu \mathrm{M}$ NBDHis mixture in the PVA+PAA-coated microchannels. Coating polymer concentrations, $0.99 \%$ PVA $+0.01 \%$ PAA. Other conditions are as in Fig. 3. 
coating was employed since the slowest migration time was obtained (Table 5), which would be advantageous for the zone electrophoretic separation in a short effective length of the microchannel. As shown in Fig. 6, partially-separated peaks were observed with the SEFs of 83 87 (Table 6). Electrophoretic resolution $\left(R_{\mathrm{S}}\right)$ of NBD-HA and His was estimated to be 0.71 . This insufficient resolution would be due to a short separation length, only $2.5 \mathrm{~cm}$. When a longer channel (normally $5.5 \mathrm{~cm}[23,31]$ ) microchip is employed for the LVSEP analysis, the resolution will be improved. In the present stage, furthermore, the RSDs were also poor as demonstrated in Tables 5 and 6. To achieve accurate and precise analyses, internal standards should be included in the sample solution.

\section{Conclusions}

To realize the LVSEP analysis of cations in MCE, in this study, the vacuum-drying method was applied to immobilize cationic polymers onto the PDMS-glass hybrid microchannel for reversal EOF. In the PB-coated channel, poor enrichments and efficiencies were obtained mainly due to a slower $\mu_{\mathrm{eo}, \mathrm{S}}$, whereas narrower peaks and better SEFs were gained in the PVA+PAA coating with a faster EOF in the stacking step. Furthermore, HA and His could be separated with the SEFs of 83 87 in MCE-LVSEP. By employing the developed approach, sensitive analyses of various cationic compounds including biogenic amines, amino acids, metal complexes, inorganic cations, and so on, are expected in MCE [35].

\section{Acknowledgment}

This work was supported by JSPS KAKENHI Grant Numbers JP15K05527 and JP18K05162. This research was also supported by SENTAN, JST.

\section{References}

[1] Castro, E. R.; Manz, A. J. Chromatogr. A 2015, 1382, 66-85.

[2] Breadmore, M. C. J. Chromatogr. A 2012, 1221, 42-55.

[3] Burgi, D. S.; Chien, R. L. Anal. Chem. 1991, 63, 20422047.

[4] Burgi, D. S.; Chien, R. L. Anal. Biochem. 1992, 202, 306-309.

[5] He, Y.; Lee, H. K. Anal. Chem. 1999, 71, 995-1001.

[6] Quirino, J. P.; Terabe, S. Science 1998, 282, 465-468.

[7] Quirino, J. P.; Terabe, S.; Otsuka, K.; Vincent, J. B.; Vigh, G. J. Chromatogr. A 1999, 838, 3-10.

[8] Isoo, K.; Terabe, S. Anal. Chem. 2003, 75, 6789-6798.

[9] Kenndler, E. J. Chromatogr. A 2014, 1335, 16-30.

[10] Kitagawa, F.; Nojiri, M.; Sueyoshi, K.; Otsuka, K. Chromatography 2014, 35, 125-129.

[11] Kitagawa, F.; Matsuo, A.; Sueyoshi, K.; Otsuka, K. Chromatography 2017, 38, 38-43.
[12] Britz-McKibbin, P.; Chen, D. D. Y. Anal. Chem. 2000, $72,1242-1252$.

[13] Timerbaev, A. R.; Hirokawa, T. Electrophoresis 2006, 27, 323-340.

[14] Hirokawa, T.; Koshimidzu, E.; Xu, Z. Electrophoresis 2008, 29, 3786-3793.

[15] Xu, Z.; Nakamura, K.; Timerbaev, A. R.; Hirokawa, T. Anal. Chem. 2011, 83, 398-401.

[16] Šlampová, A.; Malá, Z.; Gebauer, P.; Boček, P. Electrophoresis 2017, 38, 20-32.

[17] Breadmore, M. C.; Wuethrich, A.; Li, F.; Phung, S. C.; Kalsoom, U.; Cabot, J. M.; Tehranirokh, M.; Shallan, A. I.; Abdul Keyon, A. S.; See, H. H.; Gaspar, A.; Dawod, M.; Quirino, J. P. Electrophoresis 2017, 38, 33-59.

[18] Breadmore, M. C.; Abdul Keyon, A. S.; Shallan, A. I.; Dawod, M.; Quirino, J. P. Electrophoresis 2013, 34, 29-54.

[19] Kitagawa, F.; Kawai, T.; Otsuka, K. Anal. Sci. 2013, 29, 1129-1139.

[20] Kitagawa, F.; Otsuka, K. J. Chromatogr. A. 2014, 1335, 43-60.

[21] Kitagawa, F.; Osanai, O.; Nukatsuka, I. Chromatography 2019, 40, 79-82.

[22] Kitagawa, F.; Wakagi, S.; Takegawa, Y.; Nukatsuka, I. Anal. Sci. 2019, 35, 889-893.

[23] Kitagawa, F.; Ishiguro, T.; Tateyama, M.; Nukatsuka, I.; Sueyoshi, K.; Kawai, T.; Otsuka, K. Electrophoresis 2017, 38, 2075-2080.

[24] Kitagawa, F.; Kinami, S.; Takegawa, Y.; Nukatsuka, I.; Sueyoshi, K.; Kawai, T.; Otsuka, K. Electrophoresis 2017, 38, 380-386.

[25] Kawai, T.; Ueda, M.; Fukushima, Y.; Sueyoshi, K.; Kitagawa, F.; Otsuka, K. Electrophoresis 2013, 34, 2303-2310.

[26] Kawai, T.; Ito, J.; Sueyoshi, K.; Kitagawa, F.; Otsuka, K. J. Chromatogr. A 2012, 1267, 65-73.

[27] Kawai, T.; Koino, H.; Sueyoshi, K.; Kitagawa, F.; Otsuka, K. J. Chromatogr. A 2012, 1246, 28-34.

[28] Kawai, T.; Watanabe, M.; Sueyoshi, K.; Kitagawa, F.; Otsuka, K. J. Chromatogr. A 2012, 1232, 52-58.

[29] Kawai, T.; Sueyoshi, K.; Kitagawa, F.; Otsuka, K. Anal. Chem. 2010, 82, 6504-6511.

[30] Kitagawa, F.; Kawai, T.; Sueyoshi, K.; Otsuka, K. Anal. Sci. 2012, 28, 85-93.

[31] Kitagawa, F.; Nakagawara, S.; Nukatsuka, I.; Hori, Y.; Sueyoshi, K.; Otsuka, K. Anal. Sci. 2015, 31, 11711175.

[32] Miyano, H.; Toyo'oka, T.; Imai, K. Anal. Chim. Acta 1985, 170, 81-87.

[33] Kitagawa, F.; Kubota, K.; Sueyoshi, K.; Otsuka, K. Sci. Technol. Adv. Mater. 2006, 7, 558-565.

[34] Xu, Y.; Jiang, H.; Wang, E. Electrophoresis 2007, 28, 
Chromatography 2021, 42, 109-114

4597-4605.

[35] Kitagawa, F. Anal. Sci. 2020, 36, 899-900. 